\title{
Zivilurteile als Disziplinierungsmittel
}

Mit dem Urteil gegen Horst Mahler ${ }^{1}$ erreicht die justizielle Verfolgung einer politischen Opposition und die damit intendierte Liquidation eine neue Qualität. Neben den circa is ooo Strafverfahren handelt es sich heute bei dem Zivilrechtsverfahren gegen Mahler längst nicht mehr um einen Einzelfall. In Köln soll der Asta-Vorsitzende Laepple für die Schäden anläßlich der Straßenbahndemonstrationen haften. Die Frankfurter Sozietätsdruckerei hat den SDS auf 71000 DM Schadenersatz verklagt. Kieler Studenten sollen für Schäden einer Institutsbesetzung einstehen. Die in Strafverfahren Abgeurteilten werden mit einer Fülle von Zivilrechtsverfahren rechnen müssen. Während Strafprozesse durch ihre Dauer die psychische Mürbemachung der Betroffenen beabsichtigen, sollen Zivilrechtsverfahren die bürgerliche Existenzzerstörung bezwedken. Politische Apathie soll die Folge sein, die man sich in unserem Staate wünscht.

Das Mahler-Urteil zeigt auf der methodologischen Ebene und von seiner juristischen Diktion her, wie politische Sachverhalte ihres politischen Inhalts entkleidet werden, damit Recht überhaupt erst funktional werden kann, zeigt in sehr einprägsamer Weise die politischen Vorstellungen der Rechtsprechenden, nämlich Gesellschaft als hierarchisch strukturiertes Gewaltverhältnis, politische Opposition als Gefahrenquelle per se.

$I$.

Das Gericht geht davon aus, daß Mahler das Eigentum der Firma Axel Springer u. Sohn und Ullstein GmbH fahrlässig verletzt habe, und zwar »durch Vernachlässigung der erforderlichen Sorgfalt. « Der Beweis dieser Behauptung erfolgt methodisch auf drei Stufen: Die erste Stufe ist angefüllt mit einer einzigen politischen Phrase, die zweite befiehlt juristisch-abstrakte Verhaltensnormen und die dritre Stufe schließlich spielt das große Theater von der Subsumtion. Ausgangspunkt aller juristischen Argumentation ist folgende Uberlegung der ersten Stufe. Hier heiße es: Die Anwendung von Gewalt ist wals Mittel der politischen Auseinandersetzung in jedem Falle rechtswidrig, als Mittel der politischen Beeinflussung ... sogar schädlich; denn durch Gewalt läße sich niemand überzeugen.« In diesem einen Satz wird so getan, als habe es in der Geschichre niemals eine $\mathrm{Ge}$ waltdiskussion gegeben; daß Gewalt sehr wohl ein Mittel zur Befreiung des Menschen aus Unterdrüdkung und Verknechtung darstellen kann, daß es Unterschiede progressiver und reaktionärer Gewalt gibt, scheint diesen Richtern niemals zu Bewußtsein gekommen zu sein. Sie scheinen niemals revolutionäre $\mathrm{Ge}$ walt in Beziehung gesetzt zu haben zu der Vernichtung von Menschen in imperialistischen Kriegen. Hierbei geht es nicht um die Rechrfertigung von Gewalt, sondern um die Feststellung von Tatsachen. Aber hinter der Ablehnung von $\mathrm{Ge}-$ walt verbirgt sich noch etwas anderes: Hier wird der politische Sachverhalt, warum überhaupt eine Demonstration gegen den Springer-Konzern stattfand, aus den Urreilsgründen herauskatapultiert. Die Schüsse auf Rudi Dutschke hatten ihre Ursache in der beispieilosen Hetze dieses Konzerns gegen die außerparlamentarische Opposition. In der ${ } \mathrm{BZ}$ " vom I1. 2. 1968 zum Beispiel hieß es:

1 LG Berlin KJ 1969, S. 293 ff. 
„Mit scharfen Worten ist heure kein Ende zu bereiten. Da muß man hart und unerbitrlich mit solchen Burschen umgehen. Auch eine Demokratic hat ihre Gesetze, deren richrige Anwendung abschreckend wirken kann! Tatenloses Zusehen grenzt an Feigheit vor richtiger Demokratie." Oder die BZ vom 8. 2. 1968 : - Wenn wir zur Selbsthilfe greifen müssen: ich bin dabei die Ordnung wieder herzustellen. “ Die verfassungsmäßig gewährleistete Demonstrationsfreihcit gerinnt in den Urteilsgründen zur schlichten Feststellung: »Sorgfalt« schuldet der Demonstrant »auch demjenigen, gegen den demonstriert wird. « Damit hat man sich der ganzen Problematik der verfassungsrechtlichen Durchdringung des Zivilrechts entledigt, d. h. Verfassungsgrundsätze cinfach liquidierr.

Ist nunmehr der Sachverhalt glcichsam entpolitisicrt, kann auf der zwciten Stufe die Konstruktion scheinbar wertneutraler Verhaltensregulierung beginnen. Die aufgestellten Normen sind zwar in keinem Gesetzestext zu finden, aber sic haben eine Logik in sich selbst, denn wer könnte daran zweifeln: "Wer eine Gefährdung verursachr, ist verpflichter, die zur Gefahrenabwehr erforderlichen Maßnahmen zu treffen." Wer aber - trotz Gefährdung - nicht Abstand nimmr, der handelt "schuldhaft und ist gemäß $\ 823$ BGB zum Schadencrsatz verpflichtet." Nur, an dieser Stelle weiß es schon jeder, Mahler ist zum Schadenersatz verpflichrer, denn (und das bringr dann der dritre subsumtive Teil in einem dramatischen Ringen um die »korrektwissenschaftliche Subsumtion ") er hat nicht Abstand genommen. Schon von der ökonomischen Anlage des Urteils her - der dritte subsumtive Teil ist fünffach so lang wie der erste und zweite Teil zusammen - ist zu sehen, daß man sich überflüssigerweise mit etwas beschäftigt, was doch schon längst feststeht. Da den Normen, von denen sich das Gerichr »leiten ließ «, eine Rationalität fehlt, - es handelt sich um Thesen I) ... 2) ... 3)... können sie in ihrer Abstrakcheit für jedes Ergebnis, das benötige wird, fungibel gemacht werden, hier dafür, daß Mahler verurteilt wird. Den dritten, subsumtiven Teil härte sich das Gericht sparen können, da es schon das Ergebnis wußte.

II.

Inhaltlich versucht das Urteil eine Verkehrssicherungspflicht für jeden Beteiligten einer Demonstration zu konstruieren, denn es bestehe »die Rechtspflicht zu einer sachgemäßen Organisation der Demonstration $\alpha$, da von Demonstrationen immer Gefährdungen ausgehen, ohne darzulegen, was denn überhaupt gefährdet wird. Gerade die Auseinandersetzung mit den Objekten, die gefährdet werden, häıte die Pflicht zu verfassungsmäßiger Interpretation auferlegt, sei es der Gefährdung des Straßenverkehrs, des Eigentums oder des Rechts am eingerichteten und ausgeübren Gewerbebetrieb. Daß Demonstrationen Gefährdungen mit sicri bringen, wird damit begründet, daß sich Demonstranten sin großer Erregung * befinden. *Das Gefühl, das eigene Wollen durch die vielen anderen Demonstranten verfielfältigt zu sehen, war - wie sich schon an den Sprechchortexten ergab - gecignet, Hemmungen gegenüber Unrechrshandlungen abzubauen «, und außerdem wird genannt: die "Gefahr, die von emotional beeinflußsten Demonstranten und von den sich unter sie mischenden Rockers ausgeht.\& An dieser Stelle werden die Teilnehmer einer Demonstration als ein Haufen unmündiger, nicht denkender Individuen dargestelit, die beliebig dirigierbar seien, so wie es ihre Führer woilen. Die Teilnahme des Volkes am politischen Willensbildungsprozeß 3 , wie sie grandgesetzlich vorgesehen ist, scheint einem BewtBrsein, das in den kollektiven 
Handlungen politisch denkender Menschen eine gefährliche Ansammlung hysterischer Schwachköpfe sieht, nicht bekannt zu sein. Hinter dem faschistoiden Versuch, ein Versammein von Menschen als Gefahr abzustempein, liegt der konstrukrive Versuch, für Demonstranten eine Gefährdungshaftung zu konstruieren, wie sie dem Bürgerlichen Gesetzbuch außer den gesetzlich festgelegten Tatbeständen fremd ist. Ein Beispiel der geserzlich geregelten Gefährdungshaftung ist der $\$ \$_{33}$ BGB, die Tierhalterhaftung. Die Gefährlichkeir eines Tieres und die totale Abhängigkeit von Menschen begründen die Haftung des Tierhalters. Wenn in den Urteilsgründen eine quasi-Gefährdungshaftung erdichtet wird, dann kann - man hieran Gedankengänge verfolgen, die Strauß ein paar Monare später in Worte faßre.

Die Kausalitärsfrage scheint für das Gericht ganz ohne Probleme gewesen zu sein. Immerhin wird in den abstrakten Voraussetzungen noch von der Norwendigkeit der Gefahrverursachung gesprochen. Weiter unten heißt es »Urheber der Gefahr ... ist ein jeder, der in irgendeiner Weise an dem Zustandekommen der Demonstration beteiligt ist. « Die bloße Teilnahme genügr; denn ohne die Teilnahme der Demonstranten ist eine Demonstration nicht denkbar. Entweder handelt es sich hier um eine neue Kausalitätstheorie, denn sogar nach der viel weiteren Äquivalenztheorie wäre Mahler nicht heranzuziehen, denn auch ohne ihn wäre die Demonstration abgelaufen, oder es handelr sich bei den Ausführungen des Gerichts um eine Kollekrivhaftung (Voraussetzung Singularis: Urheber ist jeder, in der Begründung plötzlich Pluralis: denn ohne die Demonstranten), die es aber nicht gibr. Im Zusammenhang mit Rechtspflichten liegt Kausalität dann vor, wenn pflichtgemäßes Handein das schädigende Ereignis mit Sicherheit vereirelı hätre. Auch in dieser Beziehung läßr sich eine Kausalität für Mahler nur dann begründen, wenn - ohne davon zu sprechen - man seinen Einfluß auf die Steuerung von Demonstranten für so wesentlich hält, daß bei seinem Eingreifen alles anders abgelaufen wäre. Es wird über das nebensächilich erscheinende Problem der Kausalität die Rädelsführertheorie nun auch im Zivilrecht geboren. Die politischen Inhalte der außerparlamentarischen Opposition, unter anderem das antiautoritäre Element, widersprechen gerade der Rädelsführertheorie. Gleichwohl operieren mit solchen Gedanken Polizeibeamte und Richter. Die Existenz egalitär strukturierter politischer Opposition ist für ein Bewußtsein unvorstellbar, das in den Bahnen rigider Ordnungsstrukturen und Subordinationsverhältnissen denkt und lebr: das tagtäglich erleben muß, wie diese Verhälenisse durch Manipulation der Massen versteinert werden. Die Verinnerlichung des Prinzips des verwalteten Menschen führt dazu, auch die Massen von Demonstranten als in dumpfer Passivität verharrend und durch *Rädelsführer « manipuliert zu sehen.

Nur in diesem Zusammenhang wird die juristische Argumentation des Gerichts begreifbar, "die sachgemäße Organisation der Demonstration « als Verkehrssicherungspflicht i.S. des $₫ 823$ Abs. I BGB darzustellen. Bisher geregelte Fälle der Verkehrssicherungspflicht sind z. B. die P丹licht des Veranstalters eines Autorennens, mögliche Vorkehrungen zu treffen, daß eine Gefährdung der Zuschauer verhindert wird oder die Absicherung von Gefahren, die von Gebäuden und Grundstücken ausgehen. Das alles sind Fälle, in denen sich eine mögliche Kontrolle ausüben läßr. Der Veranstalter einer Demonstration und die einzelnen Demonstranten können aber niemals eine solche Kontrolle über Menschen ausüben. Das würde einen totalen Gehorsam der einzelnen Individuen voraussetzen oder aber die totale Gewalt einzelncr über die Masse von Demonstranten, was wiederum Ausdruck der Vorstellung vom Menschen als einem ohnmächtigen, ab- 

unsere Verfassung nicht.

III.

Ober die Verkehrssicherungspflichten wird in dem Mahler-Urteil die Demonstrationsfreiheit praktisch zunichte gemacht, da die Beteiligung an einer Demonstration ohne die Gefahr einer zivilrechtlichen Haftbarmachung nicht mehr möglich ist. Daß mit einem solchen Urteil Demonstranten eingeschüchtert werden sollen und die Verhinderung der Umsetzung theoretischer Erkenntnisprozesse in politische Praxis bezweckt wird, versteht sich von selbst. Daß man sich in dem vorliegenden Fall gerade Horst Mahler herausgegriffen hat, zeigt zudem, daß es den Herrschenden ganz besonders darauf ankommt, linken Anwälten in der Bundesrepublik und auch den Genossen in anderen Berufen die Grenzen ihrer Tätigkeit aufzuzeigen.

Henry Düx

\section{Formalismus und Autorität}

Einige Anmerkungen zu neueren Aufsäızen Eberhard Schmidts

Galt Eberhard Schmidt durch seine zahlreichen Veröffentlichungen, insbesondere seinen Lehrkommentar, dem Juristen unlängst noch als Verfechter einer vor allem die Rechte des Beschuidigten und Angeklagten wahrenden Strafprozeßordnung (worauf sich Schmidt in einer Entgegnung auf die Angriffe von Puls beruft ${ }^{1}$ ), so zeigt sich in seinen nach wie vor sehr zahlreichen Publikationen der letzten $\mathrm{I}^{1 / 2} \mathrm{Jahre}$ eine erschreckende Tendenz, diese Rechte vor allem ihm politisch unliebsamen Personen gegenüber einzuschränken. Im Grunde ist darin jedoch kein Widerspruch zu sehen, weil für den historischen Liberalismus des I9. Jh., zu dem sich Eb. Schmidt in seinem Aufsatz "Strafrechtspflege in Gefahr $^{2} \propto$ eindeurig bekennt, geradezu charakteristisch ist, daß er nicht gewillt ist, denen eine gerechte Behandlung zuteil werden zu lassen, die sich an seine Spielregeln nicht halten, weil sie erkennen, daß diese Spielregeln nur der Aufrechterhaltung der bestehenden Macht- und Herrschaftsverhältnisse dienen. Der Liberale traditioneller Prägung muß dort versagen, wo es darum geht, sozialen Strömungen von unten und der Forderung nach gesellschaftlichem Fortschritr gerectit zu werden. Die Toleranz, die große Errungenschaft des liberalen Zeitalters, schlägt in der Praxis in krasseste Intoleranz um. Zur Abwehr auch nur zaghafter reformistischer Anschauungen ${ }^{3}$ verbündet sich der Liberale leicht mit denen, die

\footnotetext{
Eb. Samide NJW 69, 1018.

ZStW $80,567 \mathrm{ff}$.

S S. Eb. Sdimidt, Zur Demokratisicrung der Jusriz in: Der Staat r'ig69 S. 77: . Das Facale und Beunruhigende dieser Reformbestrcbungen ....
} 